OPEN ACCESS

Edited by:

Filippo Cieri,

Cleveland Clinic, United States

Reviewed by:

Arun Bokde,

Trinity College Dublin, Ireland

Jianhui Zhong,

University of Rochester, United States

${ }^{*}$ Correspondence:

Abbas Babajani-Feremi ababajan@uthsc.edu

${ }^{\dagger}$ Data used in preparation of this article were obtained from the

Alzheimer's Disease Neuroimaging Initiative (ADNI) database

(adni.loni.usc.edu). As such, the

investigators within the ADNI

contributed to the design and

implementation of ADNI and/or provided data but did not participate in analysis or writing of this report. A complete listing of $A D N I$ investigators can be found at: http://adni./oni.usc. edu/wp-content/uploads/ how_to_apply/

ADNI_Acknowledgment_List.pdf

Specialty section:

This article was submitted to Applied Neuroimaging

a section of the journal

Frontiers in Neurology

Received: 04 April 2019 Accepted: 05 August 2019

Published: 30 August 2019

Citation:

Hojjati SH, Ebrahimzadeh A and

Babajani-Feremi A (2019) Identification of the Early Stage of Alzheimer's Disease Using Structural

MRI and Resting-State fMRI.

Front. Neurol. 10:904

doi: 10.3389/fneur.2019.00904

\section{Identification of the Early Stage of Alzheimer's Disease Using Structural MRI and Resting-State fMRI}

\author{
Seyed Hani Hojjati ${ }^{1,2,3}$, Ata Ebrahimzadeh ${ }^{2}$ and Abbas Babajani-Feremi ${ }^{1,3,4 *}$ \\ for the Alzheimer's Disease Neuroimaging Initiative ${ }^{\dagger}$
}

\begin{abstract}
${ }^{1}$ Department of Pediatrics, University of Tennessee Health Science Center, Memphis, TN, United States, ${ }^{2}$ Department of Electrical Engineering, Babol University of Technology, Babol, Iran, ${ }^{3}$ Neuroscience Institute and Children's Foundation Research Institute, Le Bonheur Children's Hospital, Memphis, TN, United States, ${ }^{4}$ Department of Anatomy and Neurobiology, University of Tennessee Health Science Center, Memphis, TN, United States
\end{abstract}

Accurate prediction of the early stage of Alzheimer's disease (AD) is important but very challenging. The goal of this study was to utilize predictors for diagnosis conversion to AD based on integrating resting-state functional MRI (rs-fMRI) connectivity analysis and structural MRI (sMRI). We included 177 subjects in this study and aimed at identifying patients with mild cognitive impairment $(\mathrm{MCl})$ who progress to $A D, M C l$ converter ( $\mathrm{MCl}-\mathrm{C})$, patients with $\mathrm{MCl}$ who do not progress to $\mathrm{AD}, \mathrm{MCl}$ non-converter (MCl-NC), patients with $A D$, and healthy controls $(\mathrm{HC})$. The graph theory was used to characterize different aspects of the rs-fMRI brain network by calculating measures of integration and segregation. The cortical and subcortical measurements, e.g., cortical thickness, were extracted from sMRI data. The rs-fMRI graph measures were combined with the sMRI measures to construct input features of a support vector machine (SVM) and classify different groups of subjects. Two feature selection algorithms [i.e., the discriminant correlation analysis (DCA) and sequential feature collection (SFC)] were used for feature reduction and selecting a subset of optimal features. Maximum accuracy of 67 and $56 \%$ for three-group ("AD, MCl-C, and $\mathrm{MCl}-\mathrm{NC}$ " or "MCl-C, MCl-NC, and $\mathrm{HC}$ ") and four-group ("AD, MCl-C, MCl-NC, and HC") classification, respectively, were obtained with the SFC feature selection algorithm. We also identified hub nodes in the rs-fMRI brain network which were associated with the early stage of $A D$. Our results demonstrated the potential of the proposed method based on integration of the functional and structural MRI for identification of the early stage of $A D$.

Keywords: Alzheimer's disease (AD), mild cognitive impairment (MCI), resting-state fMRI, graph theory, machine learning, hub nodes

\section{INTRODUCTION}

Alzheimer's disease $(\mathrm{AD})$ is a neurodegenerative disorder, known as a disconnection syndrome that disturbs communication between different brain regions (1). It implies that the brain network is changed during the transition from healthy condition to mild cognitive impairment (MCI) and AD. Since intervention prior to the occurrence of overt and irreversible neuronal loss is critical for the maintenance of normal brain function, prediction of $\mathrm{MCI}$, and conversion to $\mathrm{AD}$ in cognitively normal older adults is a priority for $\mathrm{AD}$ research. 
Approximately 15\% of adults older than 65 years old suffer from $\mathrm{MCI}$ and from these more than half progress to $\mathrm{AD}$ within 5 years (2). Prediction of early stage of $\mathrm{AD}$ is important and several studies have focused on investigating this prediction. Structural magnetic resonance imaging (sMRI) can be utilized to reliably characterize brain volumes, areas, cortical thickness, and curvature (3), and has been widely used to investigate alteration of these brain measures in transition from normal aging to AD. Patients with AD have diminished memory and executive function. Patients with early stage $\mathrm{AD}$ can have MCI, and although impaired, may perform similarly to normal older adults on easier memory tasks. Prognostic predictions of subjects from prodromal stages such as MCI is an area of great clinical interest $(4,5)$. However, prediction of symptomatic progression remains a relatively unexplored task. Memory impairment and dementia are common in the elderly population. Prognostic forecasting of symptom severity is complicated not only by the heterogeneity in demographics and clinical presentation, but also highly variable and non-linear symptom patterns exhibited in those suffering from $\mathrm{MCI}(6,7)$. In a recent study, Eskildsen et al. used patterns of cortical thickness and identified cortical regions potentially discriminative for separating MCI patients into converters (MCI$\mathrm{C}$ ), who received a diagnosis of $\mathrm{AD}$ dementia within 2 years, and non-converters (MCI-NC), who remained stable for 3 years (8). They reported promising results for the prediction of patients with prodromal $\mathrm{AD}$ progressing to probable $\mathrm{AD}$. Eskildsen et al. considered "time to conversion" and separated patients in different groups based on this time, and reported $<80 \%$ accuracy for predicting conversion to AD. Beheshti et al. utilized a voxel based morphometric technique to extract the global and local gray matter volumes, and then used these volumes to classify AD and healthy controls (HC) (9).

Several studies have investigated AD induced alterations of the brain network using the resting-state functional MRI (rsfMRI) (10-16). rs-fMRI has been shown to be a powerful tool for identifying the pathophysiology of functional connectivity not only in patients with $\mathrm{AD}$ but also in patients with other neurological or neuropsychiatric conditions (17). Accumulating evidence suggests that intrinsic connectivity at rest provides the communication channels of task information (18). rs-fMRI networks have been shown to be highly sensitive to AD (19). We and other investigators have reported the ability of rs-fMRI in identification of patients with MCI and AD $(13-15,20)$. We demonstrated potential of rs-fMRI in prediction of the early stage of AD (10). Grieder et al. suggested that cognitive decrease symptoms in $\mathrm{AD}$ is directly related to reduction of complexity in the brain network (21). It was reported that rs-fMRI functional connectivity can show AD-related cognitive impairment in an aging population with health, MCI, and AD (16).

Neuroimaging modalities, such as positron emission tomography (PET), diffusion-tensor imaging (DTI), rs-fMRI, and sMRI, have been found informative in providing biomarkers of conversion from MCI to AD (22-27). While most of previous studies considered a single modality approach for diagnosis of $\mathrm{AD}$ (28), it is expected that a multi-modal approach can improve accuracy of prediction of conversion to AD compared to a single-modality approach (29). Tong et al. utilized features extracted from sMRI, PET, cerebrospinal fluid (CSF) biomarkers, and categorical genetic information, and classify $\mathrm{HC}, \mathrm{MCI}$ and AD with an accuracy of 60.2\% (29). Peng et al. developed a kernel-learning-based method to combine sMRI, PET, and genetic information for $\mathrm{AD}$ and $\mathrm{MCI}$ diagnosis, and reported classification accuracies of $96.1 \%, 80.3 \%$, and $76.9 \%$ for $\mathrm{AD}$ vs. HC, MCI vs. HC, and AD vs. MCI, respectively (30). Ahmed et al. combined DTI with sMRI to improve accuracy of AD, MCI and $\mathrm{HC}$ classification, and obtained an accuracy between $76 \%$ (AD vs. $\mathrm{MCI}$ ) to $90 \%$ (AD vs. $\mathrm{HC}$ ) for two-group classification. Dyrba et al. applied a multimodal approach based on sMRI, DTI, and rs-fMRI and classify AD from $\mathrm{HC}$ with an area under curve (AUC) of the receiver operating characteristic of $82 \%$ (31).

The brain topology analysis based on the graph theory provides powerful tools to study structural and functional characteristics of the brain network. Graph theory is a mathematical tool that is capable of concisely quantifying the properties of complex systems and modeling interrelationships between the brain regions. Since a large number of local and global graph measures, i.e., features, can be extracted from the brain networks, reducing dimension of features is an essential process for identifying optimal subset of features. In this study, we employed two feature selection algorithms in a machine learning approach to identify discriminative features for classifying $\mathrm{AD}, \mathrm{MCI}$, and normal aging. We developed an automatic classification algorithm that combined information from sMRI with rs-fMRI graph measures to classify four groups of subjects (AD, MCI-C, MCI-NC, and $\mathrm{HC}$ ). We used baseline rs-fMRI and sMRI data for MCI-C and MCI-NC patients. The MCI-NC patients did not convert to $\mathrm{AD}$ in 36 months after the baseline rs-fMRI, although MCI-C patients converted to AD from 6 to 36 months after the baseline rs-fMRI. We did not use the "time to conversion" of MCI-C patients in our algorithm to test performance of the proposed method in a challenging condition where this information is unknown in real clinical application. Our aim was to develop a method with an ability to distinguish potential "MCI-decliners" from those who remain stable. To our knowledge, this is the first study that investigated integration of rs-fMRI and sMRI for four-group classification (i.e., AD, MCI-C, MCI-NC and HC).

\section{MATERIALS AND METHODS Overall Procedure}

The overall procedure of our proposed method is shown in Figure 1. Structural MRI (T1-weighted images) and rs-fMRI data of 177 subjects (34 AD, 25 MCI-C, 69 MCI-NC, and 49 $\mathrm{HC})$ were used in this study. After preprocessing of rs-fMRI data, we used the Dosenbach atlas (32) to parcellate the brain into 160 region of interests (ROIs), and the adjacency matrix was calculated using the Pearson correlation between the time series of each pair of brain regions. We calculated 10 local and 13 global graph measures based on the adjacency matrix in each patient. FreeSurfer was utilized for preprocessing, cortical reconstruction, and volumetric segmentation of sMRI images. Volumes of the subcortical structures in addition to the surface area, curvature, thickness, and volume of 148 cortical areas, based 


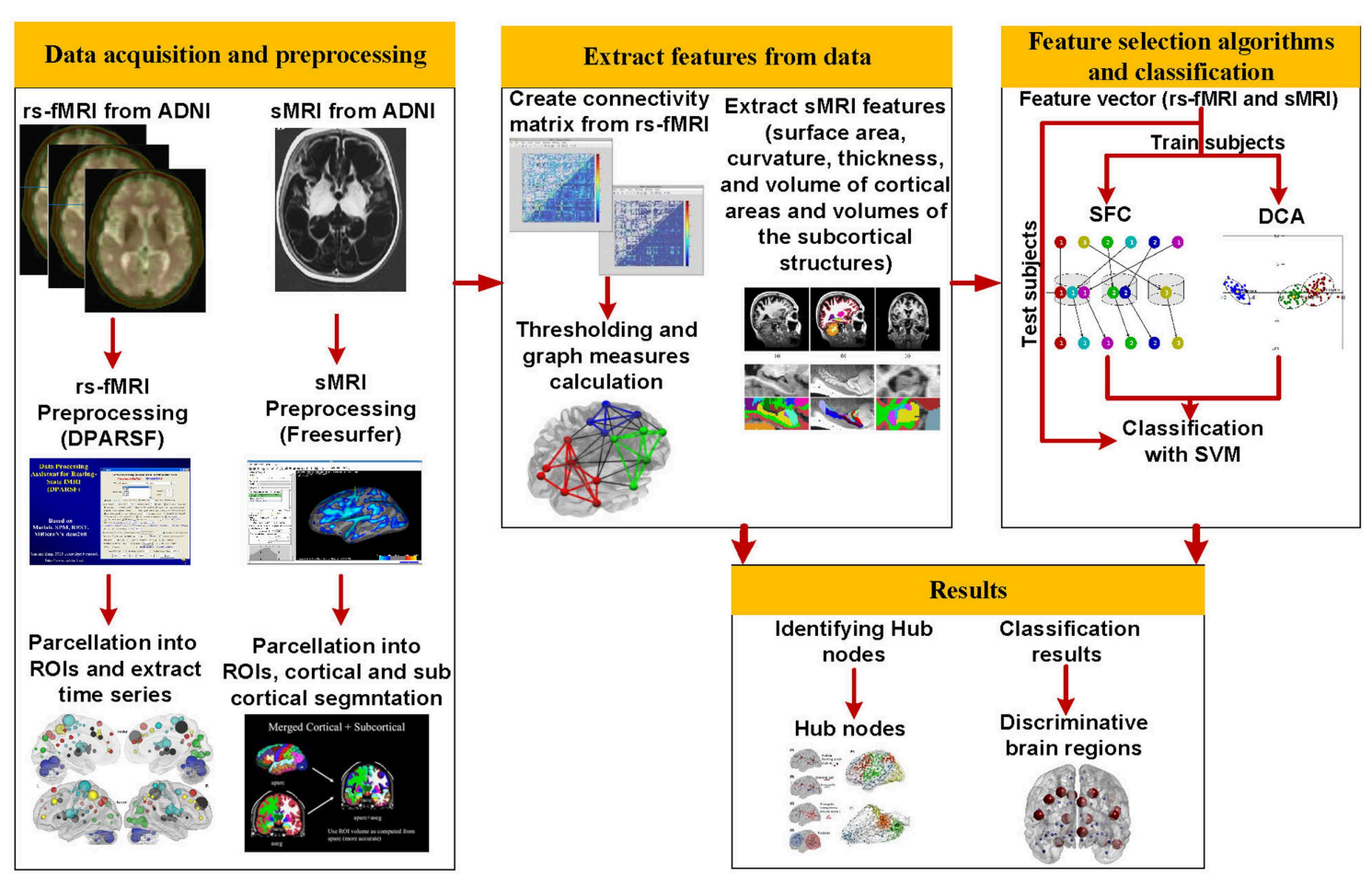

FIGURE 1 | The overall procedures of this study.

on the Destrieux atlas (33), were calculated and used as sMRI features in our algorithm. The rs-fMRI local and global graph measures were combined with sMRI measures to generate a feature vector in each patient. Two feature selection algorithms were applied to find an optimal subset of features for support vector machine (SVM) (10). We trained, cross-validated, and tested SVM to classify AD, MCI-C, MCI-NC, and HC using the selected rs-fMRI and sMRI features. We performed a second analysis on rs-fMRI data to identify hub nodes of the brain network and identify alteration of hubs in transient from healthy aging to $\mathrm{AD}$.

\section{Subjects}

We included 34 patients with $\mathrm{AD}$ (average age 72.5 years, 18 female), 25 patients with MCI-C (average age 73 years, 11 female), 69 patients with MCI-NC (average age 72.9 years, 37 female), and $49 \mathrm{HC}$ (average age 74.4 years, 28 female) from Alzheimer's Disease Neuroimaging Initiative (ADNI) database in this study. Subjects of this study were selected based on availability of both rs-fMRI and sMRI datasets in ADNI. We used the diagnosis variables to select the converted and nonconverted subjects. In the current study, we tried to include all subjects, listed in ADNI database and had a complete set of sMRI and rs-fMRI data. The MCI-C patients were converted to $\mathrm{AD}$ between 6 and 36 months. The MCI-NC patients did not convert to $\mathrm{AD}$ after 36 months of follow-up. The patients with AD had a Mini-Mental State Examination (MMSE) score of 2026, a Clinical Dementia Rating (CDR) of 0.5 or 1.0 , and met the
National Institute of Neurological and Communicative Disorders and Stroke and the $\mathrm{AD}$ and Related Disorders Association (NINCDS/ADRDA) criteria for probable AD. The patients with MCI had MMSE scores between 24 and 30, a memory complaint, objective memory loss measured by education adjusted scores on Wechsler Memory Scale Logical Memory II, a CDR of 0.5, absence of significant levels of impairment in other cognitive domains, essentially preserved activities of daily living, and an absence of dementia. The normal subjects were non-depressed, non-MCI, and non-demented, and had a MMSE score of 24-30 and a CDR close to zero. Demographic information of subjects is summarized in Table 1. Subjects for this study were selected based on availability of both rs-fMRI and sMRI datasets.

\section{Data Acquisition and Preprocessing}

The functional and structural MRI images were collected according to the ADNI acquisition protocol (34) ${ }^{1}$. A total of 140 functional volumes (TR/TE $3000 / 30 \mathrm{~ms}$, flip angle $=80^{\circ}$, $3.313 \mathrm{~mm}$ slice thickness, 48 slices) were obtained. Standard preprocessing routines were applied on rs-fMRI dataset using Data Processing Assistant for Resting State fMRI (DPARSF)

\footnotetext{
${ }^{1}$ Data used in the preparation of this article were obtained from the Alzheimer's Disease Neuroimaging Initiative (ADNI) database (adni.loni.usc.edu) that was launched in 2003 and led by Principal Investigator Michael W. Weiner, MD. The primary goal of ADNI has been to test whether serial MRI, positron emission tomography (PET), other biological markers, and clinical and neuropsychological assessment can be combined to measure the progression of mild cognitive MCI and early AD. For up-to-date information, see www.adni-info.org.
} 
TABLE 1 | Demographic and clinical information.

\begin{tabular}{|c|c|c|c|c|c|}
\hline & $\mathrm{HC}$ & MCI-NC & $\mathrm{MCl}-\mathrm{C}$ & $A D$ & $P$-value \\
\hline Number & 49 & 69 & 25 & 34 & \\
\hline Male/Female & $21 / 28$ & $32 / 37$ & $14 / 11$ & $16 / 18$ & $0.76^{a}$ \\
\hline MMSE score & $29.35 \pm 1.63$ & $27.57 \pm 2.21$ & $26.64 \pm 1.85$ & $21.24 \pm 3.37$ & $0.0003^{b}$ \\
\hline CDR score & $0.035 \pm 0.21$ & $0.5 \pm 0.0$ & $0.5 \pm 0.0$ & $0.92 \pm 0.31$ & $0.0001^{b}$ \\
\hline
\end{tabular}

MMSE, mini-mental state examination; CDR, clinical dementia rating; ${ }^{a}$ Fisher extract test; ${ }^{b}$ ANOVA test.

package (35) and SPM12 toolbox (http://www.fil.ion.ucl.ac.uk/ spm). Slice-timing correction to the last slice was performed. The fMRI time-series realigned using a six-parameter rigid-body spatial transformation to compensate for head movement effects. Then all images were normalized into the Montreal Neurological Institute (MNI) space, resampled to $3-\mathrm{mm}$ isotropic voxels, detrended, smoothed using a Gaussian filter with FWHM = $4 \mathrm{~mm}$, and band-pass filtered $(0.01-0.08 \mathrm{~Hz})$. To reduce the effect of the physiological artifacts, the whole-brain signal was removed by a multiple linear regression analysis. In addition to the global mean signal, six head motion parameters, the cerebrospinal fluid (CSF), and the white matter signals were removed as nuisance covariates to reduce the effects of motion and non-neuronal BOLD fluctuations (36).

T1-weighted MRI of all subjects were processed using FreeSurfer (version 4.5.0). Cortical reconstruction and volumetric segmentation were performed using the following procedures: removal of non-brain tissue using a hybrid watershed/surface deformation procedure (37); automated Talairach transformation; segmentation of the subcortical white matter and deep gray matter volumetric structures (including hippocampus, amygdala, caudate, putamen, and ventricles) (37-39); intensity normalization; tessellation of gray matter and white matter boundary; automated topology correction (40); and deformation following intensity gradients to optimally place the gray/white and gray/cerebrospinal fluid borders at the location where the greatest shift in intensity defines the transition to the other tissue class $(41,42)$. After completing the cortical models, registration to a spherical atlas was performed (43), followed by cortical parcellation based on Destrieux atlas (33), and subcortical segmentation.

\section{Extracting Features From rs-fMRI and sMRI Data}

An adjacency matrix was calculated using the Pearson's correlation between the time series of the fMRI signals of all pairs of 160 ROIs of Dosenbach atlas. We used a similar method detailed in our previous studies (12-15) and converted the weighted adjacency matrices to binary ones by applying an optimal threshold (44). By maximizing the global cost efficiency (GCE) of the brain network (44), we identified an optimal threshold for each adjacency matrix. To have the same number of connections after thresholding of the adjacency matrices of all subjects, we used an average optimal threshold across subjects at $19.2 \%$, and then computed the graph measures. Then 10 local and 13 global graph measures were calculated based on rs-fMRI adjacency matrix. The local graph measures were betweenness centrality, clustering coefficient, characteristic path, community structure Newman, community structure Louvain, eccentricity, eigenvector centrality, rich club coefficient, sub graph centrality, and participation coefficient (45). The global graph measures were assortativity, clustering coefficient, characteristic path, community structure Newman output, community structure Louvain output, cost efficiency (two measures), density, efficiency, graph radius, graph diameter, transitivity, and small-worldness (45).

The surface area, average cortical curvature, average and standard deviation of thickness, and volume of gray matter of 148 cortical areas (according to Destrieux atlas) were considered as sMRI features in our algorithm. We also considered 34 features corresponding to volumes of subcortical structures in our algorithm. To make measurements comparable between subjects, an anatomical normalization was performed. For each subject, all volume quantifications were divided by the corresponding estimated intracranial volume (eTIV) and area quantifications were divided by the total area of the same hemisphere. Neither cortical thickness nor curvature needed to be anatomically normalized. After the feature extracting step, the features were normalized in each subject individually.

\section{Feature Selection}

Solving pattern recognition or classification problems with data of high dimensionality is a challenging issue, particularly in neuroimaging applications with limited samples, and large number of features. The learning models tend to overfit and become less generalizable if input features are redundant or irrelevant to classification. Feature selection is usually performed to identify relevant features, reduce dimensionality of the trained model, and improve generalization of the model (12). An efficient feature selection algorithm is the essential part of a machine learning approach in case of high dimensional features. We utilized two feature selection algorithms in this study: discriminant correlation analysis (DCA) and sequential feature collection (SFC). We have shown efficiency of the SFC feature selection algorithm in identifying the early stage of $\mathrm{AD}(10,11)$. Efficiency and reliability of the DCA feature selection algorithm have also been demonstrated in several previous studies $(46,47)$.

DCA has been used in pattern recognition applications for fusing the features extracted from multiple modalities or combining different feature vectors extracted from a single 
modality (48). DCA has a low computational load and can be employed in real-time applications. DAC is a variant of principal component analysis (PCA). PCA is the most prominent tool for reducing size of a high-dimensional feature vector, especially in unsupervised learning. DCA was developed for supervised learning environment, as a supervised PCA, to maximize the discriminant capability of classification (49). The DCA transforms features space into signal and noise subspaces. The signal subspace of DCA is associated with classification effectiveness and the noise subspace is not related to the discriminant power of the classification. For a feature vector in DCA, a within-class and a between-class scatter matrices are constructed to represent the noise and signal subspaces, respectively. Then transformed features to signal space are calculated by maximizing a signal-to-noise ratio based on the within-class and between-class scatter matrices (49). The DCA can also perform an effective feature fusion by maximizing the pairwise correlations across the two feature sets and, at the same time, eliminating the between-class correlations and restricting the correlations to be within the classes.

We developed the SFC algorithm to find an optimal subset of features (with a small number of features) (10). The SFC algorithm sorts all features using the multivariate minimal redundancy maximal relevance (MRMR) feature selection algorithm. The MRMR feature selection algorithm selects features that have maximal statistical dependency based on mutual information by considering relevant and redundant features simultaneously (50). The MRMR is defined as:

$$
M R M R=M A X_{s}\left\{\frac{1}{|S|} \sum_{f_{i} \in S} I\left(f_{i} ; c\right)-\frac{1}{|S|^{2}} \sum_{f_{i}, f_{j} \in S} I\left(f_{i} ; f_{j}\right)\right\}
$$

where the relevance of a feature set $S$ for class $C$ is defined by the average value of mutual information $I(.$, .) between the individual feature $f_{i}$ and the class $C$, and the redundancy of all features in the feature set $S$ is the average value of mutual information between the features $f_{i}$ and $f_{j}$. The SFC algorithm is described in detail previously (10). Briefly, features were first sorted based on their MRMR scores. The first and the last features in the sorted feature vector had maximum and minimum discrimination ability, respectively, in classification. Then a combination of filter and wrapper feature selection algorithms were used to find optimal subset of features with best classification accuracy.

\section{Classification}

To evaluate performance of the prosed method for classification of four groups (AD, MCI-C, MCI-NC, and HC), we used the $k$-fold cross-validation (KCV) which is one of the most widely used resampling techniques (51), and its estimates for the cross-validation errors nearly agree with the true errors (52). In addition, we evaluated performance of our classification algorithm using independent and non-training test samples. To this end, we used a 5 -fold approach and assigned $80 \%$ of subjects in each of four groups to train/cross-validation set $(n=141 ; 27$ $\mathrm{AD}, 20 \mathrm{MCI}-\mathrm{C}, 55 \mathrm{MCI}-\mathrm{NC}$, and $39 \mathrm{HC}$ ) and $20 \%$ of subjects to independent and non-training test set $(n=36 ; 7$ AD, 5 MCIC, 14 MCI-NC, and $10 \mathrm{HC}$ ). We then used another 5-fold for cross-validation and further divided the train/cross-validation set to $80 \%$ for training $(n=113 ; 22 \mathrm{AD}, 16 \mathrm{MCI}-\mathrm{C}, 44 \mathrm{MCI}-\mathrm{NC}$, and $31 \mathrm{HC}$ ) and $20 \%$ for cross-validation $(n=26 ; 5$ AD, 4 MCI-C, $11 \mathrm{MCI}-\mathrm{NC}$, and $8 \mathrm{HC}$ ). We used SFC or DCA algorithms for feature selection based on a combined sMRI and rs-fMRI features of $n=141$ subjects in train/cross-validation set. An unequal sample size may cause bias in results of a classifier. We prevented this possible bias by using equal number of training and test samples in four groups based on a similar approach described in details in our previous study (11). Since MCI-C group has minimum number of subjects, we randomly selected a subset of subjects in HC, MCI-NC, and AD groups equal to the number of MCI-C subjects. This random selection was repeated 1,000 times and average performance of the classifier across this repetition was calculated.

The selected features were used to train and cross-validate an SVM to classify four groups of subjects (AD, MCI-C, MCI-NC, and HC) in the train/cross-validation set. We used SVM for classification in this study. The SVM classifier was implemented in MATLAB using LIBSVM toolbox (53). After training and cross-validating the SVM, the accuracy, sensitivity, specificity, positive predictively, and the area under curve (ROC) of the receiver operating characteristic of the trained SVM were calculated for subjects in the test sets.

\section{Hub Node Identification}

We calculated betweenness centrality and eigenvector centrality graph measures of the rs-fMRI brain network to identify hub regions. It is noteworthy that the number of hub nodes can be highly influenced by several factors, including type(s) of the centrality measure, and the value of threshold applied on the adjacency matrix. We selected the betweenness centrality and eigenvector centrality graph measures because these measures were more frequently selected by our SFC algorithm compared to other centrality measures. In addition, these measures conceptually aligned with the integrative role ascribed to hubs, as they reflect the diversity of a region's cross-network connections. Brain regions (i.e., nodes) with betweenness centrality or eigenvector centrality larger than mean plus two standard deviation across all nodes were identified as hub nodes. Since the values of graph measures depend on the level of threshold applied on the adjacency matrix, we calculated the centrality measures by using the threshold in a range from 0.1 to 0.3 with a step of 0.01 ( 21 thresholds). For each value of the threshold, the betweenness centrality and eigenvector centrality of nodes were calculated and then hub nodes were identified. Next, a percentage for identification of a node as a hub node in different threshold values was calculated. Finally, we reported hub nodes that were identified in more than $85 \%$ of thresholds.

\section{RESULTS}

\section{Three- and Four-Group Classification}

We performed three- and four-group classification using the SFC and DCA feature selection algorithms. Our results revealed that SFC outperforms DCA for feature selection in three- and four-group classification with an extra accuracy $>7 \%$ (Table 2). 
TABLE 2 | Accuracy of three- and four-group classification using the SFC and DCA feature selection algorithms.

\begin{tabular}{lll}
\hline Three-group classification & SFC & $67.6 \%$ \\
(AD, MCl-C and MCl-NC) & DCA & $57.6 \%$ \\
Three-group classification & SFC & $66.0 \%$ \\
(HC, MCl-C and MCl-NC) & DCA & $58.2 \%$ \\
Four-group classification & SFC & $56.1 \%$ \\
(AD, HC, MCl-C and MCl-NC) & DCA & $48.8 \%$
\end{tabular}

DCA, discriminant correlation analysis; SFC, sequential feature collection.

The accuracies of SVM with SFC feature selection algorithm for three-group classification ("AD, MCI-C, MCI-NC" or "MCI$\mathrm{C}, \mathrm{MCI}-\mathrm{NC}, \mathrm{HC}$ ”) and four-group classification (“AD, MCI-C, MCI-NC, HC”) were $\sim 66$ and 56\%, respectively (accuracy by chance is 33 and $25 \%$, respectively). The sensitivity, specificity, positive predictive value (PPV) and AUC of SFC algorithm are listed in Table 3, and the confusion matrix is shown in Figure 2. Our algorithm is very specific (>96\%) but not sensitive (24\%) in identifying MCI-C patients. In fact, the majority of missclassified MCI-C patients (48\%) were identified as MCI-NC, which indicates similarity of the brain network and structural abnormalities of MCI-C patients with that of MCI-NC patients. Our proposed method has a good sensitivity (62\%) and specificity (72\%) for identifying MCI-NC patients. The majority of missclassified MCI-NC patients (21\%) were identified as HC, which points to a mild abnormalities of the brain of MCI-NC patients compared to that of normal aging subjects.

\section{Important Features for Four-Group Classification}

Top features that were selected by the SFC feature selection algorithm in at least $80 \%$ of training folds were listed in Table 4 that consisted of only rs-fMRI graph measures. We, however, found that eight sMRI features, including thickness of five cortical areas, were selected by the SFC algorithm in at least $60 \%$ of training folds. The top six features listed in Table 4 correspond to the rs-fMRI graph measures and represent modularity of the brain network in six brain regions (Figure 3). We used analysis of variance (ANOVA) and found a significant between-group difference $(P<0.01)$ in three features, i.e., community structure Louvain in medial cerebellum and superior frontal cortex and community structure Newman in post occipital (Table 4). Values of these three features in $\mathrm{AD} v s$. MCI-C and $\mathrm{HC}$ vs. MC-NC are plotted in Figure 4. Results of this figure show that values of these features are clustered in $\mathrm{AD}$ and $\mathrm{HC}$ but are scattered in MCI-C and MCI-NC, indicating a similar modularity of the brain network across subjects in $\mathrm{AD}$ or $\mathrm{HC}$ group but a diverse modularity in patients with MCI corresponding to different rates of dementia in these patients.

\section{Hub Analysis}

Hub nodes of the rs-fMRI brain network in four groups of subjects (AD, MCI-C, MCI-NC, and HC) are listed in Table 5. We identified 11 nodes as the hub nodes based on two centrality measures, betweenness centrality, and eigenvector centrality, in
TABLE 3 | Sensitivity, specificity, positive predictive value (PPV), AUC, and accuracy of three- and four-group classification based on the SFC feature selection algorithm.

\begin{tabular}{|c|c|c|c|c|}
\hline & & $\begin{array}{l}\text { Three group } \\
\text { classification } \\
\text { (AD, MCl-C, } \\
\text { MCI-NC) }\end{array}$ & $\begin{array}{l}\text { Three group } \\
\text { classification } \\
\text { (MCl-C, } \\
\mathrm{MCl}-\mathrm{NC}, \\
\mathrm{HC} \text { ) }\end{array}$ & $\begin{array}{l}\text { Four group } \\
\text { classification } \\
\text { (AD, MCl-C, } \\
\text { MCl-NC, } \\
\mathrm{HC})\end{array}$ \\
\hline \multirow[t]{4}{*}{ Sensitivity (\%) } & $A D$ & 52.3 & - & 46.1 \\
\hline & $\mathrm{MCl}-\mathrm{C}$ & 36.0 & 44.0 & 24.0 \\
\hline & $\mathrm{MCl}-\mathrm{NC}$ & 89.6 & 71.7 & 61.8 \\
\hline & $\mathrm{HC}$ & - & 69.5 & 75.5 \\
\hline \multirow[t]{4}{*}{ Specificity (\%) } & $A D$ & 91.1 & - & 85.0 \\
\hline & $\mathrm{MCl}-\mathrm{C}$ & 97.7 & 90.8 & 96.1 \\
\hline & $\mathrm{MCl}-\mathrm{NC}$ & 47.5 & 74.7 & 72.0 \\
\hline & $\mathrm{HC}$ & - & 72.6 & 66.3 \\
\hline \multirow[t]{4}{*}{ PPV (\%) } & $A D$ & 77.3 & - & 49.7 \\
\hline & $\mathrm{MCl}-\mathrm{C}$ & 85 & 52.8 & 76.6 \\
\hline & $\mathrm{MCl}-\mathrm{NC}$ & 67.3 & 75.6 & 65.5 \\
\hline & $\mathrm{HC}$ & - & 63.5 & 53.5 \\
\hline \multirow[t]{4}{*}{$A \cup C$} & $A D$ & 0.72 & - & 0.65 \\
\hline & $\mathrm{MCl}-\mathrm{C}$ & 0.67 & 0.68 & 0.60 \\
\hline & $\mathrm{MCl}-\mathrm{NC}$ & 0.69 & 0.74 & 0.66 \\
\hline & $\mathrm{HC}$ & - & 0.72 & 0.70 \\
\hline \multirow[t]{4}{*}{ Accuracy (\%) } & $A D$ & 53 & - & 47 \\
\hline & $\mathrm{MCl}-\mathrm{C}$ & 36 & 44 & 24 \\
\hline & $\mathrm{MCl}-\mathrm{NC}$ & 89 & 72 & 62 \\
\hline & $\mathrm{HC}$ & - & 69 & 75 \\
\hline $\begin{array}{l}\text { Number of } \\
\text { selected }\end{array}$ & rs-fMRI & 12 & 25 & 25 \\
\hline features & sMRI & 8 & 5 & 7 \\
\hline
\end{tabular}

The average number of selected rs-fMRI and sMRI features (across 5-folds of training data) by the SFC algorithm are listed in two bottom rows.

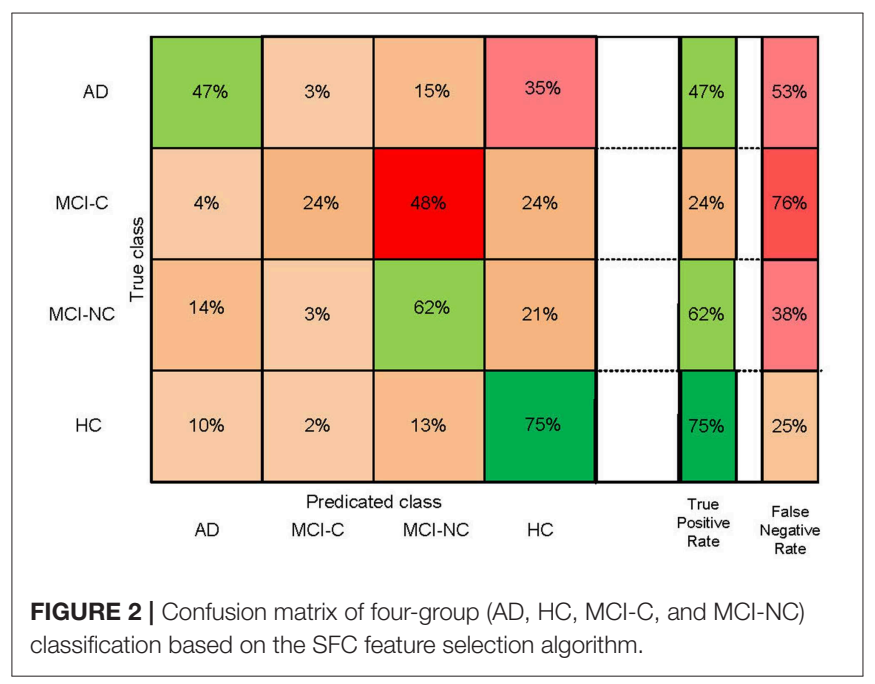

AD (6 hubs), MCI-C (5 hubs), MCI-NC (2 hubs), and HC (2 hubs). Patients with AD and MCI-C had a common hub in the basal ganglia, and this region was not a hub in MCI-NC 
and HC. MCI patients (but not AD and HC) had a hub in the parietal cortex. Insular cortex was a common hub in AD, MCI$\mathrm{C}$, and HC. On the other hand, there were group-specific hubs in each group (e.g., anterior cingulate cortex in $\mathrm{AD}$, occipital cortex in MCI-C, precentral gyrus in MCI-NC, and posterior cingulate in $\mathrm{HC}$ ) which were not identified in other groups as a hub node. It is interesting to mention that 4 out of 6 features with the most discriminant information in four-group classification (Table 4) were associated with four hub regions (i.e., insular cortex, occipital cortex, cerebellum, and precentral gyrus) listed in Table 5.

\section{DISCUSSION}

We proposed a machine learning algorithm to classify patients in the early stage of $\mathrm{AD}$ (MCI-C and MCI-NC), patients with AD, and normal aging subjects $(\mathrm{HC})$ by integrating rs-fMRI and sMRI data. This study provided three main results: (1) we examined

TABLE 4 | Top six features selected by the SFC algorithm for the four-group (AD, $\mathrm{HC}, \mathrm{MCl}-\mathrm{C}$, and $\mathrm{MCl}-\mathrm{NC}$ ) classification.

\begin{tabular}{llc}
\hline rs-fMRI graph measure & Brain area & $\boldsymbol{P}$-value \\
\hline CSL modularity & Median cerebellum & $8.7 \times 10^{-5}$ \\
CSN modularity & Post occipital & $5 \times 10^{-4}$ \\
CSL modularity & Superior frontal cortex & $8.9 \times 10^{-3}$ \\
CSN modularity & Occipital & $1.1 \times 10^{-2}$ \\
CSL modularity & Middle insula & $1.4 \times 10^{-2}$ \\
CSN modularity & Precentral gyrus & $2 \times 10^{-1}$ \\
\hline
\end{tabular}

$P$-values were calculated using the analysis of variance (ANOVA) to find a significant difference among four groups of subjects.

CSL, Community structure Louvain; CSN, Community structure Newman. the capability of integrating rs-fMRI and sMRI in a bi-modal approach to identify conversion from MCI to AD by evaluating performances of three- and four-group classifications (AD, MCI$\mathrm{C}, \mathrm{MCI}-\mathrm{NC}$, and $\mathrm{HC}$ ); (2) we evaluated performances of SFC and DCA feature selection algorithms in identification of optimal features from a large number of rs-fMRI and sMRI features. Our results revealed that the SFC algorithm outperformed the DCA feature selection algorithm by providing an extra accuracy of $>7 \%$ in four-group classification; and (3) we identified hub nodes of the rs-fMRI brain network in AD, MCI-C, MCI-NC, and HC, and found different hubs in patients within the early stage of AD.

Prediction of the early stage of $\mathrm{AD}$ using rs-fMRI and sMRI data based on a four-group classification (AD, MCI-C, MCI-NC, and HC) remains a relatively unexplored task. We performed three- and four-group classifications by integrating rs-fMRI and sMRI features, and observed that combining structural and functional MRI features improves performance of classification. We demonstrated in our previous study that a bimodal (sMRI and rs-fMRI) approach outperformed a unimodal (sMRI or rs-fMRI) approach for a two-group classification (MCI-C and MCI-NC) with an increased accuracy up to $17 \%$ (11). Therefore, we decided to use a bi-modal approach (sMRI and rs-fMRI) for three- and four-groups classification in the current study. Schouten et al. utilized functional and structural MRI and classified $16 \mathrm{AD}$ patients from 22 healthy controls, found that combining features of two modalities improves performance of classification, and achieved an accuracy of $89.5 \%$ in two-group (AD vs. HC) calcification (54). In another study, Canu et al. combined features extracted from structural MRI (cortical thickness in 68 cortical regions) and diffusion tractography (white matter microstructure) to classify 62 early onset $\mathrm{AD}$ and 27 behavioral variant of frontotemporal dementia patients, and reported $82 \%$ classification accuracy by integrating features of two modalities (55). Suk et al. integrated sMRI and
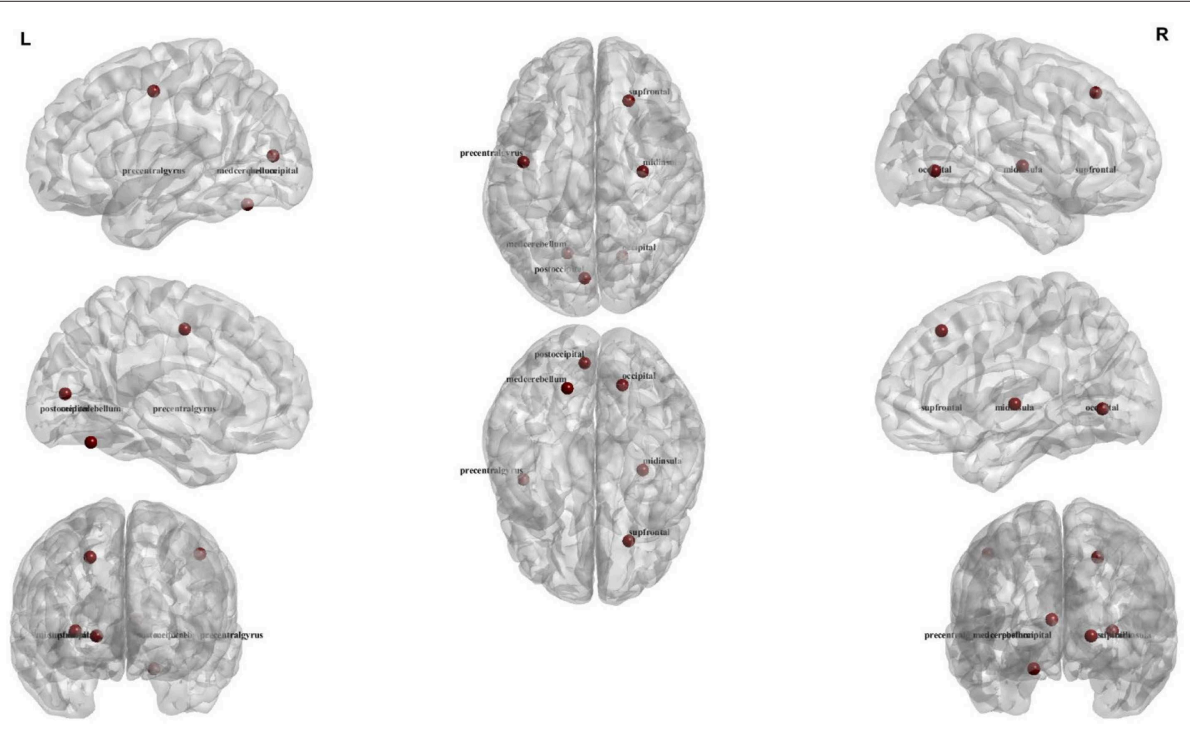

FIGURE 3 | |llustration of the six regions corresponding to top six rs-fMRI features for four-group (AD, HC, MCl-C, and MCI-NC) classification. 

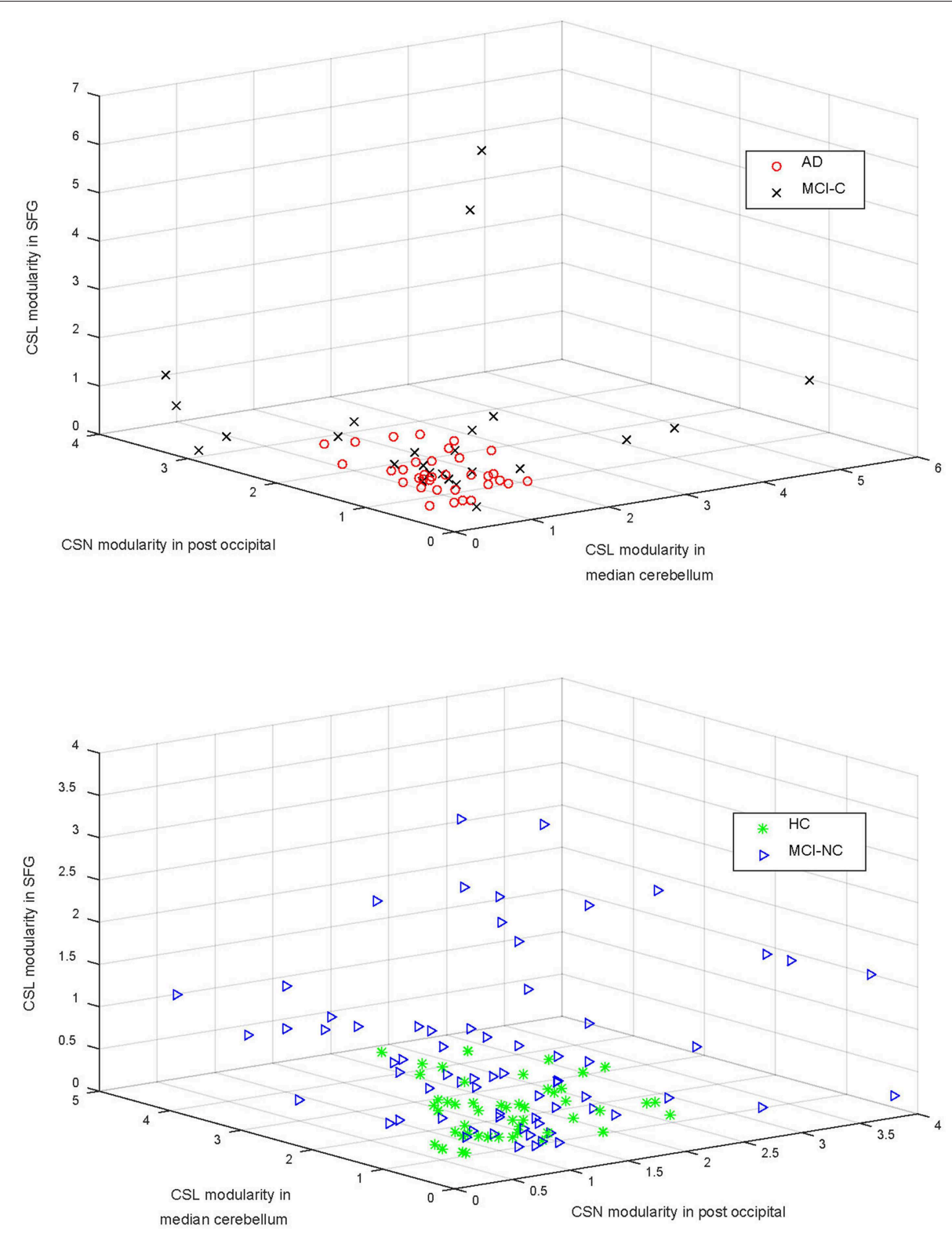

FIGURE 4 | The community structure Louvain (CSL) modularity and community structure Newman (CSN) modularity in three areas (i.e., superior frontal gyrus - SFG, median cerebellum, and post occipital cortex) are compared in the top and bottom panels for AD vs. MCl-C and HC vs. MCI-NC, respectively. Modularity of the brain network in these regions were significantly different in four groups $(P<0.01$; Table 4).

positron emission tomography (PET) features in a deep learning algorithm, and reported 98.8, 90.7, 83.7, and 83.3\% accuracies in binary (two-group) classification $\mathrm{AD} / \mathrm{HC}, \mathrm{MCI} / \mathrm{HC}, \mathrm{AD} / \mathrm{MCI}$, and MCI-C/MCI-NC, respectively (56). It is noteworthy that Suk et al. did not test performance of their proposed algorithm in three- or four-group classification, as we did. To our knowledge, this is the first study which integrated rs-fMRI with sMRI data in a four group (AD, MCI-C, MCI-NC, and HC) classification approach. For the binary classification MCI-C vs. MCI-NC, Zhang et al. (57) achieved 73.9\% accuracy by utilizing a multi-modal neuroimaging approach using FDG-PET, sMRI, and cerebrospinal fluid (CSF) data. Beheshti et al. used sMRI features in a discriminative feature ranking method to find the most discriminative feature set, and reported 75\% accuracy in MCI-C vs. MCI-NC binary calcification (9). In another study, Young et al. provided AD prediction model by adding the apolipoprotein E (ApoE) genotype to FDG-PET, sMRI, and CSF data, and reported $74 \%$ accuracy in classification of MCI-C vs. MCI-NC (58). It is noteworthy that previous studies have shown that MCI is a heterogeneous condition where MCI-NC subjects appear more healthy and MCI-C subjects appear more in AD condition (59).

Figure 2 shows that our algorithm was more accurate in identification of HC compared to other groups of subjects, which is expected due to the similarity of the brain structure and network of patients with MCI and AD compared to that of $\mathrm{HC}$. 
TABLE 5 | The rs-fMRI hub nodes in four groups of subjects (AD, HC, MCl-C, and $\mathrm{MCl}-\mathrm{NC})$.

\begin{tabular}{lcccc}
\hline Hub nodes & AD & MCI-C & MCI-NC & HC \\
\hline Basal ganglia & $X$ & $X$ & - & - \\
Temporal & $X$ & - & - & - \\
Anterior cingulate cortex (ACC) & $X$ & - & - & - \\
Medial frontal cortex (mFC) & $X$ & - & - & - \\
Thalamus & $X$ & - & - & - \\
Parietal & - & $X$ & $X$ & - \\
Insula & $X$ & $X$ & - & X \\
Cerebellum & - & $X$ & - & - \\
Occipital & - & $X$ & - & - \\
Precentral gyrus & - & - & $X$ & X \\
Posterior cingulate & - & - & - & \\
\hline
\end{tabular}

The listed areas are based on the Dosenbach atlas.

The best and worst accuracies of our algorithm were $75 \%$ for $\mathrm{HC}$ and 24\% for MCI-C groups, respectively (Figure 2). Results in Table 3 show that our algorithm was specific (>90\%) but not sensitive $(<44 \%)$ in identifying MCI-C group compared to other groups, in both three- and four-group classification. The majority of miss-classified MCI-C patients were identified as MCI-NC patients (Figure 2), indicating similarity of the rs-fMRI and sMRI features of patients in the former group with that of the latter group. On the other hand, our algorithm had a good sensitivity and specificity in identifying MCI-NC patients.

Identifying MCI-C patients is a difficult task since we used the baseline rs-fMRI and sMRI data in these patients and they converted to AD 6 to 36 months after that. In addition to using the baseline data in these patients, they had a heterogeneity in their conversion time to $\mathrm{AD}$ from 6 to 36 months. MCI$\mathrm{C}$ patients who converted to $\mathrm{AD}$ in a longer time (e.g., at 36 months) after baseline may have a similar brain network and structure at baseline compared to MCI-NC patients, who did not convert to $\mathrm{AD}$. On the other hand, brain network and structure of the MCI-C patients who converted to $\mathrm{AD}$ in a shorter time (e.g., at 6 months) may be similar to that of the $\mathrm{AD}$ patients. Furthermore, MCI-C patients were the only unstable group of patients who had a change of status from MCI to AD during 36 months follow-up. In fact, subjects in HC, MCI-NC, and $\mathrm{AD}$ groups were stable and did not convert to another group during at least 36 months. Moreover, we observed instability in the MCI-C patients that some of them had conversion to $\mathrm{AD}$ and then revision to MCI during 36 months follow-up. It is noteworthy that we excluded MCI-C patients with multiple conversion and revision in our analysis. Therefore, it is expected that a classifier has a lower performance in identifying MCI$\mathrm{C}$ compared to other groups. In line with this expectation and as shown in Figure 2 and Table 3, our proposed algorithm provided a superior performance in classifying HC, MCI-NC, and $\mathrm{AD}$ compared to MCI-C. It is noteworthy that we evaluated our algorithm in identification of the early stage of $\mathrm{AD}$ based on three- and four-group classification (AD, MCI-C, MCI-NC, and $\mathrm{HC}$ ), while most previous studied investigated a two-group classification (e.g., $\mathrm{AD}$ vs. $\mathrm{HC}$ and $\mathrm{AD}$ vs. $\mathrm{MCI})(5,60,61)$.
Top six features and brain regions that were selected by SFC in four-group classification are listed in Table 4 and Figure 3. These features were all related to rs-fMRI graph measures and represent modularity of the brain network in six cortical regions (median cerebellum, post occipital, superior frontal gyrus, occipital, middle insula, and precentral gyrus). The modularity was calculated based on the community structure Louvain or community structure Newman graph measures. A modular network has an arrangement of nodes in large modules such that maximum possible number of edges lies within groups and minimum possible number of edges lies between groups (62). Modularity of the brain network has been showed to be informative in the early stages of the neurodegenerative disease (63). A recent study demonstrated the ability of modularity of the brain network in discriminating $\mathrm{AD}$ and $\mathrm{HC}$ (63). Results of ANOVA in Table 4 show that the modularity in three brain regions, i.e., median cerebellum, post occipital and superior frontal gyrus, were significantly different in four groups of subjects $(P<0.01)$. In agreement with our results, previous studies reported association of posterior occipital, superior frontal cortex, occipital, and middle insula with AD (64-66). Another observation from Table 4 is that there was no sMRI feature among the top six features which may indicate that rs-fMRI is more informative than sMRI in identification of the early stage of $\mathrm{AD}$. This observation was in agreement with previous studies reported that biomarkers based on the functional brain network may outperform biomarkers based on the structural measures in predicting the early stage of $\operatorname{AD}(11,67)$.

In Figure 4, we compared four groups based on modularity of the brain network in three regions (i.e., median cerebellum, post occipital, and superior frontal gyrus) which were significantly different in four groups $(P<0.01)$. Figure 4 shows that modularity in $\mathrm{AD}$ or $\mathrm{HC}$ was clustered across subjects but the modularity in MCI-C or MCI-NC was scattered across subjects. This scatter of modularity in patients with MCI may be related to the inhomogeneity in MCI group, as a transitional state between normal aging and $\mathrm{AD}$, in that characteristic of their brain may vary between two extremes from $\mathrm{HC}$ to $\mathrm{AD}$.

Results of our hub analyses in Table $\mathbf{5}$ are generally consistent with previous findings in terms of localization, and provide additional support for the underlying topological organization of the early stage of AD. Interestingly, some hub areas in Table 5 (i.e., insular cortex, occipital, cerebellum, and precentral gyrus) were also identified by the SFC algorithm as important areas in classification of four groups. We found that the insular cortex was a common hub node in $\mathrm{AD}, \mathrm{MCI}-\mathrm{C}$, and $\mathrm{HC}$, and our finding is in agreement with previous studies showing importance of this area in $\mathrm{AD}(68,69)$. Our finding that Basal ganglia was a common hub in AD and MCI-C is in agreement with a recent study showing pivotal role of this area in patients with earlyonset $\mathrm{AD}$ (70). We found the parietal cortex as a common hub in MCI-C and MCI-NC patients. In agreement with our finding, association of the parietal cortex with $\mathrm{AD}$ has been reported in previous studies $(71,72)$. Our results revealed that $A D$ and MCI-C subjects had larger number of hub nodes compared to MCI-NC and HC subjects which may relate to the abnormalities 
in largescale network connectivity in $\mathrm{AD}$ and MCI-C brain regions (73).

\section{DATA AVAILABILITY}

Publicly available datasets were analyzed in this study. This data can be found here: http://adni.loni.usc.edu/.

\section{ETHICS STATEMENT}

Data used in this paper were obtained from the Alzheimer's Disease Neuroimaging Initiative (ADNI) database (http://ADNI. loni.usc.edu). Data collection and sharing for this project was funded by the Alzheimer's Disease Neuroimaging Initiative (ADNI) (National Institutes of Health Grant U01 AG024904).

\section{AUTHOR CONTRIBUTIONS}

SH: preparing required data from ADNI, data analysis, statistical analysis, and drafting and revision of the manuscript. AE: interpreting results and revision of the manuscript. AB-F: study design and conceptualization, data analysis and interpretation of the results, drafting, and revision of the manuscript.

\section{FUNDING}

This study was funded by Le Bonheur Children's Hospital, the Children's Foundation Research Institute, and the Le Bonheur

\section{REFERENCES}

1. Serrano-Pozo A, FrAosch MP, Masliah E, Hyman BT. Neuropathological alterations in Alzheimer disease. Cold Spring Harb Perspect Med. (2011) 1:a006189. doi: 10.1101/cshperspect.a006189

2. Farlow M. Treatment of mild cognitive impairment (MCI). Curr Alzheimer Res. (2009) 6:362-7. doi: 10.2174/1567205097889 29282

3. He Y, Chen Z, Evans A. Structural insights into aberrant topological patterns of large-scale cortical networks in Alzheimer's disease. J Neurosci. (2008) 28:4756-66. doi: 10.1523/JNEUROSCI.0141-08.2008

4. Huang L, Jin Y, Gao Y, Thung KH, Shen D. Longitudinal clinical score prediction in Alzheimer's disease with soft-split sparse regression based random forest. Neurobiol Aging. (2016) 46:180-91. doi: 10.1016/j.neurobiolaging.2016.07.005

5. Moradi E, Pepe A, Gaser C, Huttunen H, Tohka JI. Alzheimer's disease neuroimaging, machine learning framework for early MRI-based Alzheimer's conversion prediction in MCI subjects. Neuroimage. (2015) 104:398-412. doi: 10.1016/j.neuroimage.2014.10.002

6. Jack CR Jr, Vemuri P, Wiste HJ, Weigand SD, Lesnick TG, Lowe V, et al. Shapes of the trajectories of 5 major biomarkers of Alzheimer disease. Arch Neurol. (2012) 69:856-67. doi: 10.1001/archneurol.2011.3405

7. Sabuncu MR, Desikan RS, Sepulcre J, Yeo BT, Liu H, Schmansky NJ, et al. The dynamics of cortical and hippocampal atrophy in Alzheimer disease. Arch Neurol. (2011) 68:1040-8. doi: 10.1001/archneurol.2011.167

8. Eskildsen SF, Coupé P, García-Lorenzo D, Fonov V, Pruessner JC, Collins DL, et al. Prediction of Alzheimer's disease in subjects with mild cognitive impairment from the ADNI cohort using patterns of cortical thinning, Neuroimage. (2013) 65:511-21. doi: 10.1016/j.neuroimage.2012.09.058

9. Beheshti I, Demirel H, Matsuda H, Alzheimer's Disease Neuroimaging Initiative. Classification of Alzheimer's disease and prediction of mild cognitive impairment-to-Alzheimer's conversion from structural magnetic
Associate Board, Memphis, TN. Data collection and sharing for this project was funded by the Alzheimer's Disease Neuroimaging Initiative (ADNI) (National Institutes of Health Grant U01 AG024904). ADNI was funded by the National Institute on Aging, the National Institute of Biomedical Imaging and Bioengineering, and through generous contributions from the following: AbbVie, Alzheimer's Association; Alzheimer's Drug Discovery Foundation; Araclon Biotech; BioClinica, Inc.; Biogen; Bristol-Myers Squibb Company; CereSpir, Inc.; Eisai Inc.; Elan Pharmaceuticals, Inc.; Eli Lilly and Company; EuroImmun; F. Hoffmann-La Roche Ltd and its affiliated company Genentech, Inc.; Fujirebio; GE Healthcare; IXICO Ltd.; Janssen Alzheimer Immunotherapy Research \& Development, LLC.; Johnson \& Johnson Pharmaceutical Research \& Development LLC.; Lumosity; Lundbeck; Merck \& Co., Inc.; Meso Scale Diagnostics, LLC.; NeuroRx Research; Neurotrack Technologies; Novartis Pharmaceuticals Corporation; Pfizer Inc.; Piramal Imaging; Servier; Takeda Pharmaceutical Company; and Transition Therapeutics. The Canadian Institutes of Health Research is providing funds to support ADNI clinical sites in Canada. Private sector contributions are facilitated by the Foundation for the National Institutes of Health (www.fnih.org). The grantee organization is the Northern California Institute for Research and Education, and the study is coordinated by the Alzheimer's Disease Cooperative Study at the University of California, San Diego. ADNI data are disseminated by the Laboratory for Neuro Imaging at the University of Southern California. resource imaging using feature ranking and a genetic algorithm. Comput Biol Med. (2017) 83:109-19. doi: 10.1016/j.compbiomed.2017.02.011

10. Hojjati SH, Ebrahimzadeh A, Khazaee A, Babajani-Feremi AI. Alzheimer's disease neuroimaging, predicting conversion from $\mathrm{MCI}$ to $\mathrm{AD}$ using restingstate fMRI, graph theoretical approach and SVM. J Neurosci Methods. (2017) 282:69-80. doi: 10.1016/j.jneumeth.2017.03.006

11. Hojjati SH, Ebrahimzadeh A, Khazaee A, Babajani-Feremi AI. Alzheimer's disease neuroimaging, predicting conversion from $\mathrm{MCI}$ to $\mathrm{AD}$ by integrating rs-fMRI and structural MRI. Comput Biol Med. (2018) 102:30-9. doi: 10.1016/j.compbiomed.2018.09.004

12. Khazaee A, Ebrahimzadeh A, Babajani-Feremi A. Identifying patients with Alzheimer's disease using resting-state fMRI and graph theory. Clin Neurophysiol. (2015) 126:2132-41. doi: 10.1016/j.clinph.2015.02.060

13. Khazaee A, Ebrahimzadeh A, Babajani-Feremi A. Application of pattern recognition and graph theoretical approaches to analysis of brain network in Alzheimer's disease. J Med Imag Health Informat. (2015) 5:1145-155. doi: 10.1166/jmihi.2015.1513

14. Khazaee A, Ebrahimzadeh A, Babajani-Feremi A. Application of advanced machine learning methods on resting-state fMRI network for identification of mild cognitive impairment and Alzheimer's disease. Brain Imaging Behav. (2016) 10:799-817. doi: 10.1007/s11682-015-9448-7

15. Khazaee A, Ebrahimzadeh A, Babajani-Feremi AI. Alzheimer's disease neuroimaging, classification of patients with MCI and AD from healthy controls using directed graph measures of resting-state fMRI. Behav Brain Res. (2017) 322:339-50. doi: 10.1016/j.bbr.2016.06.043

16. Lin Q, Rosenberg MD, Yoo K, Hsu TW, O'Connell TP, Chun MM. Resting-state functional connectivity predicts cognitive impairment related to Alzheimer's disease. Front Aging Neurosci. (2018) 10:94. doi: 10.3389/fnagi.2018.00094

17. Greicius M. Resting-state functional connectivity in neuropsychiatric disorders. Curr Opin Neurol. (2008) 21:424-30. doi: 10.1097/WCO.0b013e328306f2c5 
18. Ito T, Kulkarni KR, Schultz DH, Mill RD, Chen RH, Solomyak LI, et al. Cognitive task information is transferred between brain regions via resting-state network topology. Nature Commun. (2017) 8:1027. doi: 10.1038/s41467-017-01000-w

19. Sheline YI, Morris JC, Snyder AZ, Price JL, Yan Z, D’Angelo G, et al. APOE4 allele disrupts resting state fMRI connectivity in the absence of amyloid plaques or decreased CSF A 342 . J Neurosci. (2010) 30:17035-40. doi: 10.1523/JNEUROSCI.3987-10.2010

20. Stam CJ, Jones BF, Nolte G, Breakspear M, Scheltens P. Small-world networks and functional connectivity in Alzheimer's disease. Cereb Cortex. (2007) 17:92-9. doi: 10.1093/cercor/bhj127

21. Grieder M, Wang DJJ, Dierks T, Wahlund LO, Jann K. Default mode network complexity and cognitive decline in mild Alzheimer's disease. Front Neurosci. (2018) 12:770. doi: 10.3389/fnins.2018.00770

22. Binnewijzend MA, Schoonheim MM, Sanz-Arigita E, Wink AM, van der Flier WM, Tolboom N, et al. Resting-state fMRI changes in Alzheimer's disease and mild cognitive impairment. Neurobiol Aging. (2012) 33:2018-28. doi: 10.1016/j.neurobiolaging.2011.07.003

23. Bosch B, Arenaza-Urquijo EM, Rami L, Sala-Llonch R, Junqué C, SoléPadullés C, et al. Multiple DTI index analysis in normal aging, amnestic MCI and AD. Relationship with neuropsychological performance. Neurobiol Aging. (2012) 33:61-74. doi: 10.1016/j.neurobiolaging.2010.02.004

24. Lindemer ER, Salat DH, Smith EE, Nguyen K, Fischl B, Greve DN, et al. White matter signal abnormality quality differentiates mild cognitive impairment that converts to Alzheimer's disease from nonconverters. Neurobiol Aging. (2015) 36:2447-57. doi: 10.1016/j.neurobiolaging.2015.05.011

25. Okello A, Koivunen J, Edison P, Archer H, Turkheimer FK. Någren $\mathrm{K}$, et al. Conversion of amyloid positive and negative MCI to AD over 3 years: an 11C-PIB PET study. Neurology. (2009) 73:754-60. doi: 10.1212/WNL.0b013e3181b23564

26. Pagani M, Giuliani A, Oberg J, Chincarini A, Morbelli S, Brugnolo A, et al. Predicting the transition from normal aging to Alzheimer's disease: a statistical mechanistic evaluation of FDG-PET data. Neuroimage. (2016) 141:282-90. doi: 10.1016/j.neuroimage.2016.07.043

27. Risacher SL, Saykin AJ, Wes JD, Shen L, Firpi HA, McDonald BC. Baseline MRI predictors of conversion from MCI to probable $\mathrm{AD}$ in the ADNI cohort. Curr Alzheimer Res. (2009) 6:347-61. doi: 10.2174/156720509788 929273

28. Mateos-Perez JM, Dadar M, Lacalle-Aurioles M, Iturria-Medina Y, Zeighami Y, Evans AC. Structural neuroimaging as clinical predictor: a review of machine learning applications. Neuroimage Clin. (2018) 20:506-22. doi: 10.1016/j.nicl.2018.08.019

29. Tong T, Gray K, Gao QQ, Chen L, Rueckert D, Initia AD N. Multi-modal classification of Alzheimer's disease using nonlinear graph fusion. Pattern Recogn. (2017) 63:171-81. doi: 10.1016/j.patcog.2016.10.009

30. Peng JL, Zhu XF, Wang Y, An L, Shen DG. Structured sparsity regularized multiple kernel learning for Alzheimer's disease diagnosis. Pattern Recogn. (2019) 88:370-82. doi: 10.1016/j.patcog.2018.11.027

31. Dyrba M, Grothe M, Kirste T, Teipel SJ. Multimodal analysis of functional and structural disconnection in Alzheimer's disease using multiple kernel SVM. Hum Brain Mapp. (2015) 36:2118-31. doi: 10.1002/hbm.22759

32. Dosenbach NU F, Nardos B, Cohen AL, Fair DA, Power JD, Church JA, et al. Prediction of individual brain maturity using Fmri. Science. (2010) 329:1358-61. doi: 10.1126/science.1194144

33. Fischl B, van der Kouwe A, Destrieux C, Halgren E, Segonne F, Salat DH, et al. Automatically parcellating the human cerebral cortex. Cerebral Cortex. (2004) 14:11-22. doi: 10.1093/cercor/bhg087

34. Jack CR Jr, Bernstein MA, Fox NC, Thompson P, Alexander G, Harvey $\mathrm{D}$, et al. The Alzheimer's Disease Neuroimaging Initiative (ADNI): MRI methods. J Magn Reson Imaging. (2008) 27:685-91. doi: 10.1002/jmri. 21049

35. Chao-Gan Y, Yu-Feng Z. DPARSF: a MATLAB toolbox for pipeline data analysis of resting-state fMRI. Front Syst Neurosci. (2010) 4:13. doi: 10.3389/fnsys.2010.00013

36. Greicius MD, Krasnow B, Reiss AL, Menon V. Functional connectivity in the resting brain: a network analysis of the default mode hypothesis. Proc Natl Acad Sci USA. (2003) 100:253-8. doi: 10.1073/pnas.01350 58100
37. Segonne F, Dale AM, Busa E, Glessner M, Salat D, Hahn HK, et al. A hybrid approach to the skull stripping problem in MRI. Neuroimage. (2004) 22:1060-75. doi: 10.1016/j.neuroimage.2004.03.032

38. Fischl B, Salat DH, Busa E, Albert M, Dieterich M, Haselgrove $\mathrm{C}$, et al. Whole brain segmentation: automated labeling of neuroanatomical structures in the human brain. Neuron. (2002) 33:341-55. doi: 10.1016/S0896-6273(02)00569-X

39. Fischl B, Salat DH, van der Kouwe AJ, Makris N, Segonne F, Quinn BT, et al. Sequence-independent segmentation of magnetic resonance images. Neuroimage. (2004) 23(Suppl. 1):S69-84. doi: 10.1016/j.neuroimage.2004.07.016

40. Fischl B, Liu A, Dale AM. Automated manifold surgery: constructing geometrically accurate and topologically correct models of the human cerebral cortex. IEEE Trans Med Imaging. (2001) 20:70-80. doi: 10.1109/42.906426

41. Fischl B, Dale AM. Measuring the thickness of the human cerebral cortex from magnetic resonance images. Proc Natl Acad Sci USA. (2000) 97:11050-5. doi: 10.1073/pnas.200033797

42. Dale AM, Fischl B, Sereno MI. Cortical surface-based analysis. Segmentation I, surface reconstruction. Neuroimage. (1999) 9:179-94. doi: 10.1006/nimg.1998.0395

43. Fischl B, Sereno MI, Dale AM. Cortical surface-based analysis. II: inflation, flattening, a surface-based coordinate system. Neuroimage. (1999) 9:195-207. doi: 10.1006/nimg.1998.0396

44. Bassett DS, Bullmore ET, Meyer-Lindenberg A, Apud JA, Weinberger DR, Coppola R. Cognitive fitness of cost-efficient brain functional networks. Proc Natl Acad Sci USA. (2009) 106:11747-52. doi: 10.1073/pnas.0903641106

45. Rubinov M, Sporns O. Complex network measures of brain connectivity: uses and interpretations. Neuroimage. (2010) 52:1059-69. doi: 10.1016/j.neuroimage.2009.10.003

46. Soleymani S, Dabouei A, Kazemi H, Dawson J, Nasrabadi NM. Multi-level feature abstraction from convolutional neural networks for multimodal biometric identification. In: 2018 24th International Conference on Pattern Recognition (ICPR). (Beijing) (2018), 3469-76. doi: 10.1109/ICPR.2018.8545061

47. Boyaci E, Sert M. Video classification based on ConvNet collaboration and feature selection. In: 2017 25th Signal Processing and Communications Applications Conference (SIU). (Antalya) (2017), 1-4. doi: 10.1109/SIU.2017.7960515

48. Haghighat M, Abdel-Mottaleb M, Alhalabi W. Discriminant correlation analysis: real-time feature level fusion for multimodal biometric recognition. IEEE Trans Informat Forens Secur. (2016) 11:1984-96. doi: 10.1109/TIFS.2016.2569061

49. Kung SY, Chanyaswad T, Chang JM, Wu PY. Collaborative PCA/DCA learning methods for compressive privacy. In: Acm Transactions on Embedded Computing Systems (New York, NY: ACM) (2017). doi: 10.1145/2996460

50. Yang J, Zhu Z, He S, Ji Z. Minimal-redundancy-maximal-relevance feature selection using different relevance measures for omics data classification. In: Computational Intelligence in Bioinformatics and Computational Biology (CIBCB) (Singapore: IEEE) (2013). p. 246-51. doi: 10.1109/CIBCB.2013.6595417

51. Anguita D, Ghio A, Ridella S, Sterpi D. K-fold cross validation for error rate estimate in support vector machines. in: Proceedings of The 2009 International Conference on Data Mining, DMIN (Las Vegas, NV) (2009). p. 291-97.

52. Braga-Neto UM, Dougherty ER. Is cross-validation valid for smallsample microarray classification? Bioinformatics. (2004) 20:374-80. doi: 10.1093/bioinformatics/btg419

53. Chang CC, Lin CJ. LIBSVM: a library for support vector machines. ACM Trans Intell Syst Technol. (2011) 2:27. doi: 10.1145/1961189.1961199

54. Schouten TM, Koini M, de Vos F, Seiler S, van der Grond J, Lechner A, et al. Combining anatomical, diffusion, resting state functional magnetic resonance imaging for individual classification of mild and moderate Alzheimer's disease. NeuroImage Clin. (2016) 11:46-51. doi: 10.1016/j.nicl.2016.01.002

55. Canu E, Agosta F, Mandic-Stojmenovic G, Stojkovic T, Stefanova E, Inuggi A, et al. Multiparametric MRI to distinguish early onset alzheimer's disease and behavioural variant of frontotemporal dementia. Neuroimage Clin. (2017) 15:428-38. doi: 10.1016/j.nicl.2017.05.018

56. Suk HI, Lee SW, Shen D, The Alzheimer's Disease Neuroimaging Initiative. Latent feature representation with stacked auto-encoder 
for AD/MCI diagnosis. Brain Struct Funct. (2015) 220:841-59. doi: 10.1007/s00429-013-0687-3

57. Zhang D, Shen DI. Alzheimer's Disease Neuroimaging. Multi-modal multi-task learning for joint prediction of multiple regression and classification variables in Alzheimer's disease. Neuroimage. (2012) 59:895-907. doi: 10.1016/j.neuroimage.2011.09.069

58. Young J, Modat M, Cardoso MJ, Mendelson A, Cash D, Ourselin S, et al. Accurate multimodal probabilistic prediction of conversion to Alzheimer's disease in patients with mild cognitive impairment, Neuroimage Clin. (2013) 2:735-45. doi: 10.1016/j.nicl.2013.05.004

59. Cheng B, Liu M, Zhang D, Munsell BC, Shen D. Domain transfer learning for MCI conversion prediction. IEEE Trans Biomed Eng. (2015) 62:1805-17. doi: 10.1109/TBME.2015.2404809

60. Misra C, Fan Y, Davatzikos C. Baseline and longitudinal patterns of brain atrophy in MCI patients, their use in prediction of short-term conversion to AD: results from ADNI. Neuroimage. (2009) 44:1415-22. doi: 10.1016/j.neuroimage.2008.10.031

61. Wolz R, Julkunen V, Koikkalainen J, Niskanen E, Zhang DP, Rueckert $\mathrm{D}$, et al. Multi-method analysis of MRI images in early diagnostics of Alzheimer's disease. PLoS ONE. (2011) 6:e25446. doi: 10.1371/journal.pone.0 025446

62. Rubinov M, Sporns O. Weight-conserving characterization of complex functional brain networks. Neuroimage. (2011) 56:2068-79. doi: 10.1016/j.neuroimage.2011.03.069

63. Jalili M. Graph theoretical analysis of Alzheimer's disease: discrimination of $\mathrm{AD}$ patients from healthy subjects. Informat Sci. (2017) 384:145-56. doi: 10.1016/j.ins.2016.08.047

64. Arnold SE, Hyman BT, Flory J, Damasio AR, Van Hoesen GW. The topographical and neuroanatomical distribution of neurofibrillary tangles and neuritic plaques in the cerebral cortex of patients with Alzheimer's disease. Cerebral Cortex. (1991) 1:103-16. doi: 10.1093/cercor/1.1.103

65. Bai F, Xie C, Yuan Y, Shi Y, Zhang Z. Promoter haplotypes of interleukin-10 gene linked to cortex plasticity in subjects with risk of Alzheimer's disease. Neuroimage Clin. (2018) 17:587-95. doi: 10.1016/j.nicl.2017.11.019

66. Chen B, Zhong X, Mai N, Peng Q, Wu Z, Ouyang C, et al. Cognitive impairment and structural abnormalities in late life depression with olfactory identification impairment: an Alzheimer's disease-like pattern. Int J Neuropsychopharmacol. (2018) 21:640-48. doi: 10.1093/ijnp/pyy016
67. Coleman P, Federoff H, Kurlan R. A focus on the synapse for neuroprotection in Alzheimer disease and other dementias. Neurology. (2004) 63:1155-162. doi: 10.1212/01.WNL.0000140626.48118.0A

68. Davatzikos C, Bhatt P, Shaw LM, Batmanghelich KN, Trojanowski JQ. Prediction of MCI to AD conversion, via MRI, CSF biomarkers, pattern classification. Neurobiol Aging. (2011) 32:2322.e19-2322.e27. doi: 10.1016/j.neurobiolaging.2010.05.023

69. Bechdolf A, Wood SJ, Nelson B, Velakoulis D, Yucel M, Takahashi T, et al. Amygdala and insula volumes prior to illness onset in bipolar disorder: a magnetic resonance imaging study. Psychiatry Res. (2012) 201:34-9. doi: 10.1016/j.pscychresns.2011.06.010

70. McGough EL, Kelly VE, Weaver KE, Logsdon RG, McCurry SM, Pike $\mathrm{KC}$, et al. Limbic and basal ganglia neuroanatomical correlates of gait and executive function: older adults with mild cognitive impairment and intact cognition. Am J Phys Med Rehabil. (2018) 97:229-35. doi: 10.1097/PHM.0000000000000881

71. Abu-Akel A, Shamay-Tsoory S. Neuroanatomical and neurochemical bases of theory of mind. Neuropsychologia. (2011) 49:2971-84. doi: 10.1016/j.neuropsychologia.2011.07.012

72. Li R, Wu X, Fleisher AS, Reiman EM, Chen K, Yao L. Attention-related networks in Alzheimer's disease: a resting functional MRI study. Hum Brain Mapp. (2012) 33:1076-88. doi: 10.1002/hbm.21269

73. Tijms BM, Wink AM, de Haan WW, van der Flier M, Stam CJ Scheltens $\mathrm{P}$, et al. Alzheimer's disease: connecting findings from graph theoretical studies of brain networks. Neurobiol Aging. (2013) 34:2023-36. doi: 10.1016/j.neurobiolaging.2013.02.020

Conflict of Interest Statement: The authors declare that the research was conducted in the absence of any commercial or financial relationships that could be construed as a potential conflict of interest.

Copyright (๑ 2019 Hojjati, Ebrahimzadeh and Babajani-Feremi. This is an openaccess article distributed under the terms of the Creative Commons Attribution License (CC BY). The use, distribution or reproduction in other forums is permitted, provided the original author(s) and the copyright owner(s) are credited and that the original publication in this journal is cited, in accordance with accepted academic practice. No use, distribution or reproduction is permitted which does not comply with these terms. 